\title{
AC 2012-3231: CHARACTERIZATION OF STUDENT MODELING IN AN INDUSTRIALLY SITUATED VIRTUAL LABORATORY
}

\section{Erick Jacob Nefcy, Oregon State University}

Erick Nefcy is a doctoral student in the School of Chemical, Biological, and Environmental Engineering at Oregon State University. Through his undergraduate studies, he has held multiple internships at Intel Corporation. He is currently studying the growth of self-forming barrier layers in copper thin films, as well as investigating the student teams' use of models during completion of the Chemical Vapor Deposition Virtual Laboratory project.

\section{Dr. Edith Stanley Gummer, Education Northwest \\ Dr. Milo Koretsky, Oregon State University}

Milo Koretsky is a professor of chemical engineering at Oregon State University. He currently has research activity in areas related to thin film materials processing and engineering education. He is interested in integrating technology into effective educational practices and in promoting the use of higher level cognitive skills in engineering problem solving. Koretsky is a six-time Intel Faculty Fellow and has won awards for his work in engineering education at the university and national levels. 


\section{Characterization of Student Modeling in an Industrially Situated Virtual Laboratory}

\section{Introduction}

Modeling has been identified as a critical element of engineering practice. Theories regarding modeling by STEM professionals in practice contend that models are initially constructed from prior knowledge and newly gathered information and that they are refined in an iterative cycle of creation, use, evaluation, and revision. ${ }^{1,2,3}$ However, authentic modeling practices are difficult to replicate in the school environment.

In order to develop these iterative modeling skills in students we have designed the Virtual CVD Laboratory Project. It has been specifically designed to provide students an authentic, industrially-situated task which they can solve using the fundamental knowledge and skills that they have learned as undergraduates. This learning system utilizes virtual laboratories that are industrially situated where student teams take on the role of process development engineers. They are tasked with finding suitable input parameters to a process so that it can be released to high volume manufacturing. They complete the task though experimentation, but the experiments are completed virtually. When students interact with the virtual equipment, there is lower cognitive and haptic demand required to perform experiments than would be needed if working with physical equipment. This aspect affords students the opportunity to build a rich experimental design based on interpretation and iteration. ${ }^{4}$ While not explicitly instructed to do so, most student teams inevitably resort to modeling as a tool to progress towards a solution.

The study presented in this paper focuses on the Virtual CVD Laboratory Project, in which student teams are tasked with developing a 'recipe' of input parameter values for a chemical vapor deposition (CVD) reactor. The reactor deposits thin films of silicon nitride on polished silicon wafers, an initial step in the manufacture of transistors. The computer simulation generates data for film thicknesses at the wafer locations students choose to measure based on their specified input parameters. The film thicknesses incorporate random and systematic process and measurement error and are representative of an industrial reactor. The student teams must use results from successive runs to iteratively guide their solution. They are also encouraged to apply sound engineering methods as they have a limited budget and they are charged virtual money for each reactor run and each measurement. Student teams continue until they have found a 'recipe' of input parameter values that they believe yields optimal reactor performance. This project is complex and participants typically spend between 15 and 25 hours to complete the project. More information may be found in Reference 5.

Our previous work describes the development of the methodology used to create a graphical representation of student teams' model development process throughout this project. ${ }^{6}$ These graphical representations are termed Model Development and Usage Representations, or Model 
Maps, for short. The result of the previous study showed a significant difference in the modeling characteristics in the Virtual CVD Laboratory Project when compared to two capstone physical laboratories. Based on the analysis of work products (design notebooks, design memoranda and final reports) of 14 teams, the Virtual CVD Laboratory Project showed significant increases both in the number of model components students used and the variety of those model components from team to team.

However, we have been concerned that the characterization of the number and types of model components is under articulated given the relatively static nature of the data. The study reported in this paper uses the same analysis framework, but at a finer grain size using "think-aloud" protocol analysis.

The following research questions frame the study:

1. How do the Model Maps created using the coarser grain analysis based on work products compare to the finer grain analysis based on protocol data?

2. Do the coarser grain data give a reasonable representation of a team's modeling process?

\section{Assessing Learning in Virtual Laboratories}

Virtual laboratories, simulations, and educational games have recently been receiving considerable attention as an alternative mode to university instructional laboratories to achieve learning. ${ }^{7,8}$ In engineering and science, the virtual laboratory is most commonly used as an alternative mode to deliver the corresponding physical laboratory by simulating a similar set of activities; we term this type an analogous virtual laboratory. In analogous virtual laboratories, systematic split designs can be conducted to assess learning in the virtual mode and compare it to learning in the physical mode. In this manner, research shows equivalent and often greater learning gains in the virtual mode. ${ }^{9-13}$ In this paper, we study an industrially-situated virtual laboratory project that has no direct analog to the physical laboratory at the university. Assessment of student learning for these industrially situated laboratories is challenging, in part because there is not an obvious comparison group for such a study. This feature has, in some cases led to the development of graphical representations in an attempt to characterize student learning. Two studies of these types of virtual laboratories are particularly relevant to this study and are described in more detail below.

The first example is Oncology Thinking Cap $^{14}$ which has been developed to provide medical students experience with designing clinical trials of an anticancer drug. Specifically the students design a Phase II trial for study of the efficacy of the drug Pittamycin in the treatment of Metastatic Breast Cancer. Like the Virtual CVD Laboratory Project, students are able to experience the iterative design process and use the results of previous runs to modify their experimental design strategy. The developers have added a Trial Design Wizard to the simulation to help scaffold the experimental design process by identifying important design parameters that need to be specified. ${ }^{15}$ The scaffolding includes four screens that contain welldefined to help provide novice users the framework of a clinical design. 
In their epistemic game, Nephrotex, Shaffer and co-workers have developed an industrially situated virtual laboratory where students are tasked with designing a kidney dialyzer based on iterative experimentation. ${ }^{7}$ This project has been delivered to first-year engineering students and is also scaffolded, involving ten 45-minute class periods and five homework assignments. This cornerstone project is designed for students as they start their studies in engineering and the student teams treat the process as a "black box" and are directed towards statistical experimental design, completing two iterative cycles. In the first cycle they look at one of the input parameters, and in the second cycle, they look at all four input parameters. In contrast, the Virtual CVD Laboratory Project is a capstone project that is designed with the intent that students integrate their engineering science knowledge from previous classes. In both projects, the student teams must evaluate the trade-offs involved in achieving the output metrics as there is no solution that maximizes all outputs. In addition to Nephrotex, Shaffer's group has developed several other epistemic games, reinforcing practice in the professions of journalism, urban planning, and graphic arts.

Like the Virtual CVD Laboratory Project, both of these examples are industrially situated and have students complete a task within the context of professional practice. In addition, they both use graphical representations to try to capture and characterize and interpret student learning.

\section{Graphical Representations to Study Leaming}

Graphical representations are emerging as a method for learning scientists to link the goals of an instructional intervention with observations of students' actions, and through this linkage to provide evidence of learning. In general, such graphical representations allow characterization and interpretation of more complex and extensive activities. They also form a holistic and complementary alternative to the statistical analyses of quantitative methods or the fine grained but discrete analyses of qualitative methods such as discourse analysis. In this paper, we seek to contribute to this growing literature by assessing the validity of a specific graphical representation, Model Maps, to provide evidence of student modeling in a complex and industrially situated project.

The use of graphical representations to understand student learning in science and engineering is an emerging area and there is a limited literature base from which to draw. A comparison of five different studies that have employed a graphical representation as central analysis method is shown in Table 1 . In a playground design problem, Atman et al. ${ }^{16}$ compared performances of first-year engineering students, advanced engineering students, and experts. Individuals were allotted a maximum of three hours to complete the design, and it was often completed in less time. The researchers coded transcripts from a verbal protocol for design activities (problem definition, information gathering, generating ideas, modeling, feasibility, evaluation, decision, communication, and other) and then produced a chronological graphical representation of these activities. The representation allowed the researchers to conclude that experts continuously transition between activities throughout the project. This characteristic of expertise could not have been identified solely from a statistical analysis or category counts. 
Table 1. Summary of different studies employing graphical representations to provide evidence of learning

\begin{tabular}{|c|c|c|c|c|c|c|}
\hline $\begin{array}{l}\text { Paper } \\
\text { cited }\end{array}$ & $\begin{array}{l}\text { Context of } \\
\text { Activity }\end{array}$ & $\begin{array}{l}\text { Name of Virtual } \\
\text { Laboratory (if } \\
\text { applicable) }\end{array}$ & $\begin{array}{l}\text { Graphical } \\
\text { Representation }\end{array}$ & Elements & $\begin{array}{l}\text { Academic } \\
\text { Level }\end{array}$ & $\begin{array}{l}\text { Participant } \\
\text { time on task }\end{array}$ \\
\hline $\begin{array}{l}\text { Atman } \\
(2007)\end{array}$ & $\begin{array}{l}\text { Playground } \\
\text { Design }\end{array}$ & N/A & & $\begin{array}{l}\text { Design } \\
\text { activities }\end{array}$ & $\begin{array}{l}\text { First-year and } \\
\text { senior } \\
\text { engineering } \\
\text { undergraduates } \\
\text { Experts }\end{array}$ & $\begin{array}{l}\text { Up to } 3 \\
\text { hours }\end{array}$ \\
\hline $\begin{array}{l}\text { Strom } \\
(2001)\end{array}$ & $\begin{array}{l}\text { Class-wide } \\
\text { mathematics } \\
\text { activity }\end{array}$ & N/A & $\begin{array}{l}\text { Directed } \\
\text { Graphs }\end{array}$ & $\begin{array}{l}\text { Conceptual, } \\
\text { procedural and } \\
\text { prior } \\
\text { knowledge }\end{array}$ & $\begin{array}{l}\text { 2nd grade } \\
\text { students }\end{array}$ & $\begin{array}{l}1 \text { hour class } \\
\text { period }\end{array}$ \\
\hline $\begin{array}{l}\text { Hmelo- } \\
\text { Silver } \\
\text { (2003) }\end{array}$ & $\begin{array}{l}\text { Clinical trials } \\
\text { for cancer } \\
\text { patients }\end{array}$ & $\begin{array}{l}\text { Ontcology } \\
\text { Thinking Cap }\end{array}$ & CORDTRA & $\begin{array}{l}\text { Coding } \\
\text { categories }\end{array}$ & $\begin{array}{l}\text { Medical } \\
\text { students }\end{array}$ & $\begin{array}{l}\text { Two to } \\
\text { three hour } \\
\text { session }\end{array}$ \\
\hline $\begin{array}{l}\text { Shaffer } \\
\text { (2009) }\end{array}$ & Kidney dialyzer & Nephrotex & $\begin{array}{l}\text { Epistemic } \\
\text { Network } \\
\text { Analysis }\end{array}$ & $\begin{array}{l}\text { skills, } \\
\text { knowledge, } \\
\text { identity, values } \\
\text { and } \\
\text { epistemology }\end{array}$ & $\begin{array}{l}\text { First-year } \\
\text { engineering } \\
\text { undergraduates }\end{array}$ & $\begin{array}{l}10 \text { hours in } \\
\text { class plus } 5 \\
\text { homework } \\
\text { assignments }\end{array}$ \\
\hline $\begin{array}{l}\text { This } \\
\text { Paper }\end{array}$ & $\begin{array}{l}\text { Process } \\
\text { development of } \\
\text { a Chemical } \\
\text { Vapor } \\
\text { Deposition } \\
\text { (CVD) Reactor }\end{array}$ & $\begin{array}{l}\text { Virtual CVD } \\
\text { Laboratory } \\
\text { Project }\end{array}$ & $\begin{array}{l}\text { Model } \\
\text { Representation } \\
\text { and Usage } \\
\text { Maps }\end{array}$ & $\begin{array}{l}\text { Quantitative } \\
\text { models, } \\
\text { qualitative } \\
\text { models, ..., } \\
\text { experimental } \\
\text { runs }\end{array}$ & $\begin{array}{l}\text { Senior } \\
\text { undergraduates }\end{array}$ & $\begin{array}{l}\text { Average of } \\
10 \text { to } 20 \\
\text { hours }\end{array}$ \\
\hline
\end{tabular}

Strom and coworkers ${ }^{17}$ developed the method of Directed Graphs to examine a 50-minute lesson in a $2^{\text {nd }}$ grade mathematics class. Directed Graphs map the interaction of coded conversational turns between conceptual, procedural, and prior knowledge. The researchers developed this representation to illustrate how these elements coordinate in the development of a mathematical argument. The structure of this representation contains a circle with 23 distinct boxes: 13 represent specific procedural actions, 5 conceptions, and 4 knowledge from prior classes. Sequentially numbered line segments connect the boxes and show the evolution of the classroom discussion. These representations are interpreted to show how the elements of mathematical knowledge - "as conceived, as performed, and as historically rooted - were coordinated" (page 764).

In order to study how different aspects of discourse relate to one another in the Oncology Thinking Cap (described above), Hmelo-Silver ${ }^{18}$ developed a graphical representation called CORDTRA (chronologically-ordered representations of discourse and tool-related activity). CORDTRA is similar in method to the graphical representation of Atman et al. ${ }^{16}$; however, in addition to data categories, speaking turns of student team members and instructors are also displayed. This representation allows investigation of the collaborative interactions of student teams believed to build knowledge. The initial study reported results from six four-student teams who spent two to three hours on the task. Since this first study, CORDTRA has been used to study other technology mediated learning environments, including STELLAR, an online 
Problem Based Learning tool for pre-service teachers ${ }^{19}$ and RepTools which allows middle school students to explore structure-behavior-function relationships in aquatic systems. ${ }^{20}$

This epistemic games developed by Shaffer and coworkers ${ }^{21}$ are designed with the intent of developing the components which define an epistemic frame. The components consist of skills, knowledge, identity, values and epistemology that align with a particular profession. In order to understand how these various components manifest and interact, they have developed a graphical representation which they term, epistemic network analysis. This methodology stems from Social Network Analysis. The records of the players within the simulation are qualitatively coded as one or more components of the epistemic frame. The coded description of a player's performance is then mapped to a set of graphical representations that provide a snapshot in time of how the students have utilized the skills, knowledge, identity, values, and epistemology of a development engineer and how these components are linked. These graphical representations can then be used to characterize and compare "learning trajectories" as participants complete the tasks.

While each is unique, the graphical representations all contain the following common characteristics. They are:

- Visual, chronological, holistic and integrative (summarizes significant features of the entire learning experience)

- Used to interpret and illustrate interactions or relations between desired elements of study

- Uniquely constructed for each research study based on instructional design and research questions to examine the understanding and performance that the researchers desire to study

- Complex to interpret, and typically hard to make methodology transparent

- Different in the granularity of analysis (scales with extent of project)

\section{Typology of Model Components}

In this study, we seek to explore the validity and utility of a graphical representation (Model Maps) in characterizing the modeling activity of student teams in the Virtual CVD Laboratory Project. Ultimately, we are interested in understanding the ways that student teams introduce and develop model components, and how those models are influenced by the experimental runs that they perform.

We use the definition of a scientific model proposed by Schwartz et al. ${ }^{22}$ that a model is "a representation that abstracts and simplifies a system by focusing on key features to explain and predict scientific phenomena". We extend this definition to the context of engineering by claiming that models allow engineers to better develop possible solutions to a design problem. Furthermore, we assert that the ability to apply and operationalize models, including making the appropriate assumptions and simplifications, and recognizing the range of their validity is a key element of engineering practice.

In our work we recognize two main types of models components: quantitative and qualitative. 


\section{Quantitative Modeling}

Quantitative modeling, also known as mathematical modeling, is broadly defined as the representation of a system through the use of mathematical equations. ${ }^{1,23,24}$ Generally, relationships which are present in the system, such as between temperature and growth rate in the Virtual CVD Laboratory Project, are used to define the properties of the quantitative model. When developed appropriately, the model can be used to make useful numerical predictions. Quantitative modeling in the virtual laboratory project can be categorized in three ways:

1. First Principles Modeling. In this case, mathematical constructs from fundamental engineering science principles are utilized in the formulation of a quantitative model. Most student teams in the Virtual CVD Laboratory Project use material balances (conservation of mass) and many also use some form of a reaction kinetics expression.

2. Statistical modeling. These models use basic statistics principles to assist in characterizing the behavior of the problem space. For example, approaches like Design of Experiments are used to determine the nature of variable interactions.

3. Empirical Modeling. This form of modeling uses empirically developed relations to aid in completing the project. Often teams apply the principles of proportionality and assume a linear relationship to make predictions based on their measured data though interpolation or extrapolation.

\section{Qualitative Modeling}

There is a wide variety of types of qualitative models, including: inscriptions, which involve the use of tables, diagrams, graphs and maps ${ }^{24}$ propositions, which is considered the smallest unit of knowledge that one can sensibly judge as true or false; and metaphors, which allow conceptual leaps across domains. ${ }^{25}$ In this body of research, we primarily observe a type of qualitative modeling which is closely related to diSessa's phenomenological-primitives (p-prims). ${ }^{26}$

DiSessa describes p-prims as "the intuitive equivalent of physical laws; they may explain other phenomena, but they are not themselves explained within the knowledge system." Basically, students will use their interpretation of reality to construct a simple relationship which may not be supported by an excepted theoretical construct. In the virtual laboratory project, this form of qualitative modeling is commonly used in student work. As students develop a more sophisticated understanding of pertinent phenomena, the "contexts of activation may migrate, expand, or contract, depending on the elements' new roles in the developing" knowledge system. In essence, students will have a better understanding of where individual p-prims fit with respect to one another as they develop and connect knowledge structures. As expertise develops, pprims will no longer be self-explanatory and will defer to more formal and complex knowledge structures. 


\section{Methods}

Sources of Data

Two different data sources, student work products and "think-aloud" protocol analysis, were used to develop the Model Maps. The intent is to compare Model Maps developed based on work products to the more fine grained but labor intensive Model Maps based on protocol analysis. While we expect the latter method to have more detail, we are interested in determining if the Model Maps of the teams' modeling processes are foundationally similar.

\section{$\underline{\text { Work Products }}$}

Student work products include their laboratory notebook, their design memoranda, and their final report. The primary source is the laboratory notebooks. The notebooks are intended to contain all ideas and notes over the course of the project and provide evidence of the models that student teams use as well as their model progression and the strategies that they consider. This source is complemented by the written assignments and the experimental records from the virtual laboratory database. These latter sources serve to confirm, explain or expand upon the notebook content.

\section{"Think-Aloud" Protocol Analysis}

Two teams, labeled Team A and Team B, were observed and audio recorded for the complete duration of the project, which represents 15.3 and 9.5 hours of recorded work, respectively. During this time, students were instructed to verbalize their thoughts but were not encouraged to describe or explain their thoughts. To develop the Model Maps, the transcripts were coded. Transcript coding requires significant personnel and expertise resources. The analyses presented here give the perspective of one coder; a second coder was not utilized and no Cohen's Kappa statistic is presented. The data should be interpreted with this methodological limitation in mind.

\section{Participants}

Student participants were from the same cohort in the first term of the senior capstone laboratory sequence at Oregon State University. This class included 27 students majoring in bioengineering, 45 students majoring in chemical engineering, and 9 students majoring in environmental engineering. These students were assembled into 27 three-student teams who all participated in two physical laboratory projects. The virtual laboratory project was administered between two physical laboratory projects. They had a choice between the three virtual laboratory projects; 15 teams worked on the Virtual CVD Laboratory Project (45 students) and the remainder worked on a different virtual laboratory project. This research was approved by the institutional review board and the results reported here are from participants that signed informed consent forms.

\section{Model Maps (Model Development and Usage Representations)}

Model Maps are used to provide a visual depiction of student modeling during the laboratory project. These maps categorize the model component type (quantitative, qualitative, statistical or empirical), their degree of utilization (operationalized, abandoned or not engaged), their correctness, and the experimental runs to which they are relevant. This information is chronologically arranged along with the experimental runs and instructor consultations to give an appropriate context to the team's work. In total, 42 Model Maps have been completed and analyzed based on student work products. ${ }^{6}$ This paper presents initial depictions of the first two teams whose solutions are characterized by Model Maps that have been developed using 
protocol analysis. A brief description of the elements contained in a Model Map is provided below; more detail is available in reference 27.

Model components are identified in student teams' work products or in the transcripts of the protocol analysis. A student researcher assembles this information and constructs the preliminary Model Map. A faculty member, who is a domain expert, then reviews and evaluates this information for accuracy and correctness. The separation of the student researcher's production of the preliminary Model Map the domain expert's review is done intentionally to ensure consistency and reliability. The two meet and discuss until consensus is reached. Features relevant to this study are highlighted below.

\section{Model Components}

Modeling components are identified when it is clear that the use of this component furthered, or intended to further, the team's progression toward a solution. Once the component is identified, a description or mathematical expression is added to the Model Map.

Table 2 displays the types of modeling components. Quantitative Model

Components, characterized by the use of mathematical expressions or reasoning, are placed inside squares, while Qualitative Model Components, characterized by descriptive or intuitive mechanisms, are placed inside circles. To further describe the fundamental nature of a model component, an ' $S$ ' or ' $E$ ' is placed inside the model component to indicate the presence of a model component which is statistical or empirical in nature, respectively.

The line which surrounds the model
Table 2: Model Component and Run Key

\begin{tabular}{|c|c|c|}
\hline & Symbol & Name and Description \\
\hline \multirow{2}{*}{ 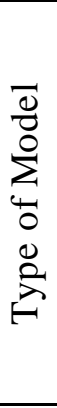 } & & $\begin{array}{l}\text { Circles represent Qualitative models, } \\
\text { relationships that do not rely on numbers, } \\
\text { e.g. "As pressure decreases, uniformity } \\
\text { increases" }\end{array}$ \\
\hline & & $\begin{array}{l}\text { Rectangles represent Quantitative } \\
\text { Models which allow teams to } \\
\text { quantitatively relate numbers (typically } \\
\text { in the form of equations), e.g. "Film } \\
\text { Deposition Rate=Reaction Rate Constant } \\
x \text { Concentration ofReactant" }\end{array}$ \\
\hline \multirow{3}{*}{ 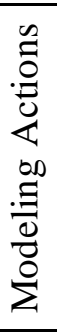 } & & $\begin{array}{l}\text { An Operationalized Model is one that is } \\
\text { developed and then used throughout the } \\
\text { solution process. }\end{array}$ \\
\hline & & $\begin{array}{l}\text { Abandoned Models are developed and } \\
\text { then clearly abandoned. }\end{array}$ \\
\hline & & $\begin{array}{l}\text { A model is classified as Not Engaged if } \\
\text { it is clearly displayed in the notebook but } \\
\text { no evidence exists of its use. }\end{array}$ \\
\hline \multirow{4}{*}{ 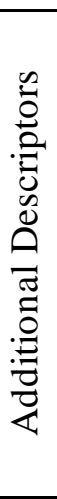 } & & $\begin{array}{l}\text { An X is placed over any Clearly } \\
\text { Incorrect Model. }\end{array}$ \\
\hline & & $\begin{array}{l}\text { A box is shown at the beginning of the } \\
\text { map, signifying the Information } \\
\text { Gathering stage. All sources listed in } \\
\text { notebook are displayed. }\end{array}$ \\
\hline & & $\begin{array}{l}\text { Primary Models are along the central } \\
\text { problem line and are used repeatedly and } \\
\text { are essential to the overall solution. }\end{array}$ \\
\hline & & $\begin{array}{l}\text { Secondary Models are connected to the } \\
\text { central problem line and are peripheral to } \\
\text { the overall solution }\end{array}$ \\
\hline \multirow{5}{*}{ 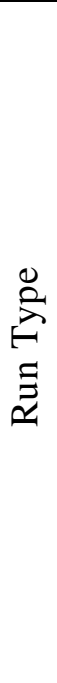 } & & $\begin{array}{l}\text { A quantitative model is used by the } \\
\text { group to determine the parameter values } \\
\text { for Model Directed Runs. }\end{array}$ \\
\hline & & $\begin{array}{l}\text { Defining parameters for a model is } \\
\text { accomplished through the use of } \\
\text { Parameter Defining Runs. }\end{array}$ \\
\hline & & $\begin{array}{l}\text { A statistical approach is used to } \\
\text { determine the parameter values for } \\
\text { Statistically Defined Runs (e.g. DOE) }\end{array}$ \\
\hline & & $\begin{array}{l}\text { Teams analyze the data provided by } \\
\text { Qualitative Verification Runs to } \\
\text { qualitatively verify models that they have } \\
\text { developed. }\end{array}$ \\
\hline & & $\begin{array}{l}\text { No explicit reason is given or deducible } \\
\text { for Runs Not Explicitly Related to } \\
\text { Modeling. Often they represent a "guess } \\
\text { and check" or a "fine tuning" run. }\end{array}$ \\
\hline
\end{tabular}
component represents the type or level of engagement associated with the particular model component. A model component surrounded by a double line represents a model which has been operationalized and retained by the team. A model component surrounded by a single line 
represents a model component which has been considered, but ultimately rejected. A team may reject a component when mathematical errors prevent its usefulness, when the data contradicts the hypothesis posed by the model, or when a more correct or relevant version of the same model component is discovered later. Model components surrounded by dashed lines are considered to be "not engaged". These model components appear where there is no evidence of utilization in mathematical work; there are no changes evident in their run parameters, or otherwise. Model components that are placed on the center line are considered central to the team's approach to the project and are designated as "Primary Model Components".

Models that lie outside the center line, connected by inward arrows are considered "Secondary Model Components", which can be seen in the second part of Table 2. Some model components are presented as groups which connect to each other vertically. These model components are considered to be "chunked" together by the student team. This formation is indicative of high level modeling.

\section{Experimental Run Markers}

Critical to the model development process in the Virtual CVD Laboratory Project is the information teams obtain from the experimental runs they complete. Experimental runs are included in the Model Maps, indicated by run markers on the center line with a corresponding run number. As shown in the bottom of Table 2, the relation between the runs and the team's model development is also identified. Separate symbols are used for: (i) Model Directed Runs (filled squares), runs where the input values have been generated by the team's model; (ii) Parameter Defining Runs (filled diamonds), runs which are used to collect data necessary to obtain a numerical value for a model parameter (e.g., activation energy); (iii) Statistically Defined Runs (filled triangles), runs which are part of a statistical design (e.g., Design of Experiments), or (iv) Qualitative Verification Runs (filled circles), runs which confirm or contradict qualitative model predictions. Experimental runs which cannot be classified in any of these categories are said to have no relation to modeling and have their own appropriate run marker. Run numbers which appear near model components indicate the relevance of that run in the development or use of that model component.

\section{Results and Discussion}

The Model Maps for Team A and Team B are presented first in the based on work products. While the original Model Maps specify particular descriptors of the model components used, the ones presented in this paper are generalized for ease of understanding. Therefore, some of the information used in the interpretations is not shown next, we present the generalized Model Maps for the same teams based on the transcripts from the "think-aloud" data. As expected, these provide greater detail, and the length of the Model Map time line expands. To provide a more direct comparison the Model Maps from the work products are also shown on a timeline of the same length. For both data sources, an explanation of the team's solution pathway will be used to highlight the similarities and differences in interpretation from each data source. 


\section{Model Maps from Work Products (Coarse Grain)}

In Figure 1A, the generic Model Map for Team A is shown. It was developed from the team's work products as discussed above. An illustration of how the Model Map can be used to interpret the major features of the team's solution. In the time spent before run 1, the team performed a literature search, identifying three references (as indicated). They initially formulated three quantitative model components, the last of which came after the design strategy meeting with the instructor. The next modeling activity occurred after run 3; the team assembled three more quantitative model components in an effort to mathematically model the virtual CVD reactor's behavior. These components were linked or chunked and became the basis for this team's approach. They used output from the resulting model to predict the input parameters in 9 of the following runs. Such use of a model is termed a "model directed run" and denoted by square run markers (runs 4, 5, 7-10,12,13,15). After run 15, the modeling behavior ceases as the team switches to a fine-tuning mode in their final 7 runs.

\section{Virtual CVD: Team A}

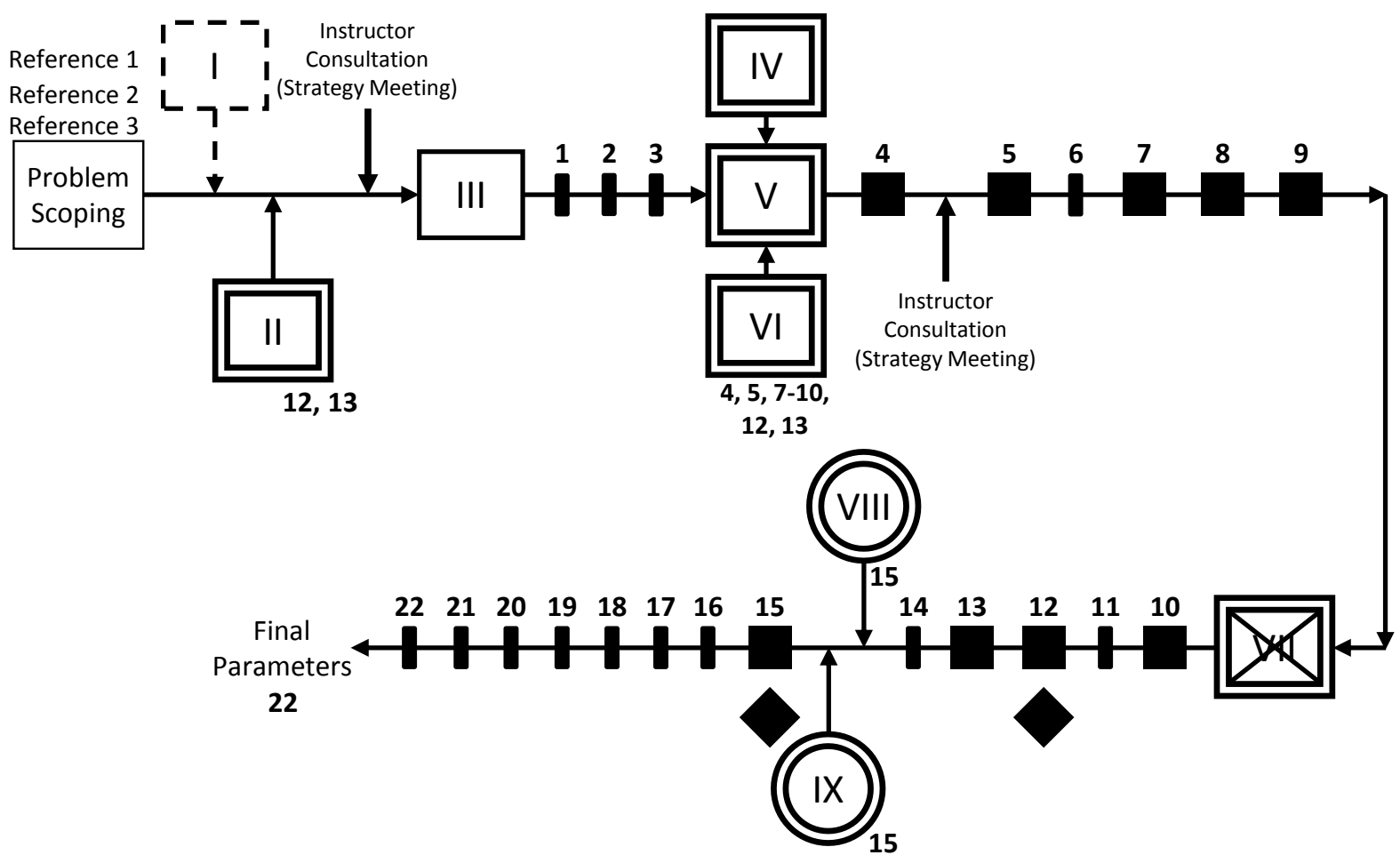

Figure 1A: The Model Map generated for Team A based on work products (coarse grain)

Team B's notebook-based Model Map is shown in Figure 1B. Much like Team A, Team B started their project with a literature search (also involving three references) and initial model component formulation. However, the characteristics of the initial modeling activity contrast from the previous team. There are many more model components ( 9 vs. 3); however most of these components are "not engaged" (as indicated by the dashed boxes) and not useful to the team in their solution. After the instructor consultation, the team began to investigate their system qualitatively, as represented by the model components X and XI which appear in the 
circles between run 1 and run 3. After Run 7 the team used a statistical methods approach, governed by qualitative model component XIII which appears as a square. This model component dominated their project from run 10 to run 21 , the runs which are represented by triangles indicating a "statistically defined run." The later runs were used to optimize their results. The final run was a repeat run, the last triangle shaped run and was used for statistical analysis for the team's final report.
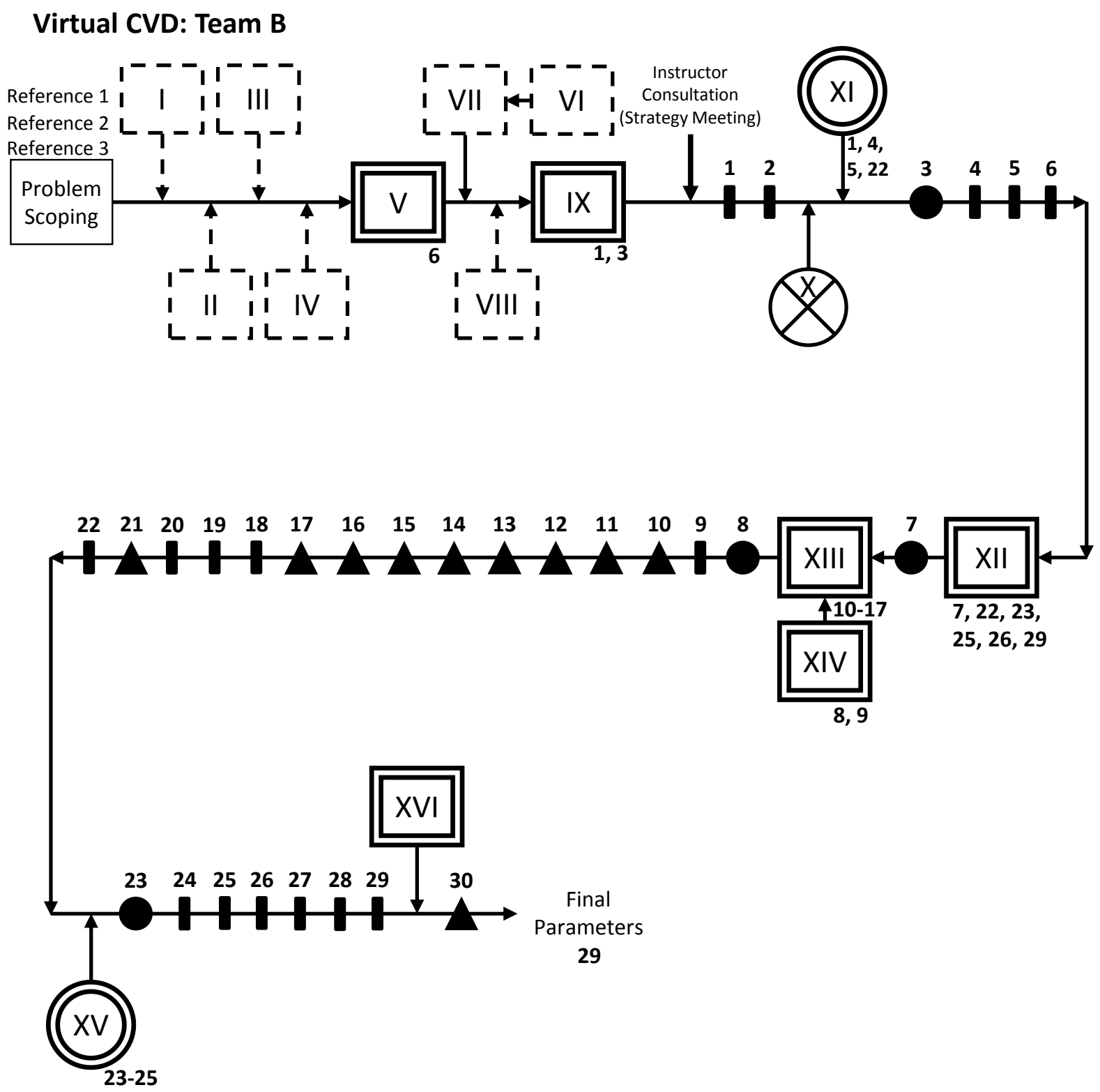

Figure 1B: The Model Map generated for Team B based on work products (coarse grain)

\section{Model Maps Protocol Analysis (Fine Grain)}

Next Model Maps that were developed from the fine grain data, the transcripts of the protocol analysis, are discussed. The model components which were also present in coarse grain Model Maps (from work products) will be highlighted via a shaded background. A classification 
scheme for how the model components compare can be seen in Figure 2. An identity change indicates a change in the model component's function (i.e. changing from abandoned to operationalized), and is depicted with a shaded grey background. Movement indicates the model component that was first utilized or considered at a different time in the team's solution through the project and is denoted with a striped grey background. Finally, a model component which remained unmoved and unchanged is denoted with a no background. In addition there are model components that appear only from the fine-grain data source. These components do not have dashed lines around them.

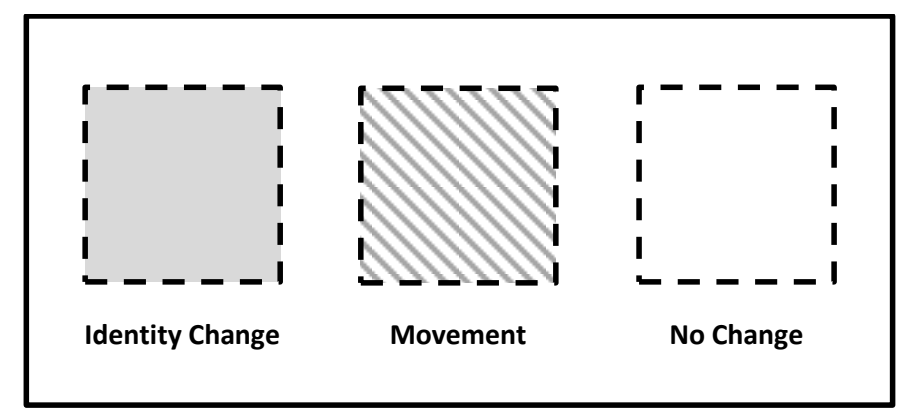

Figure 2: Protocol Analysis Model Change Key

\section{Protocol Analysis: Team A}

The Model Map from the fine grained data source for Team A is shown in Figure 3A. The components from the course grained data source (originally shown in Figure 1A) are redrawn in Figure $3 \mathrm{~B}$ with the length of the time line and the numbering changed to match Figure $3 \mathrm{~A}$.

Both data sources indicate Team A examined three references in the problem scoping phase; however the protocol analysis Model Map in Figure 5A shows a greater extent of the team's initial modeling, with the team generating fourteen model components before run 1 .

\section{The Primary Model}

A collection of three linked model components appears after run three in the course grain Model Map. As discussed above, this set of model components becomes the core of the student team's solution process, a trend which is confirmed through its utilization through run 15 . In the fine grain Model Map, more facets of the multi-component model become visible, and the result is a seven component primary model. Half of the model components that are generated during the main model's usage, through run 13 in the fine grain Model Map, are related to improving the accuracy of the main model.

\section{Fine Tuning}

A long string of "unrelated to modeling" runs are present at the end of this team's solution pathway. This behavior commonly indicates the team's use of fine tuning methods and is exhibited from run 16 through the end of the project in the coarse grain Model Map. New model components observed in the fine grain Model Map relate to the interactions between reactor parameters and measurement effects, which show the group validating that the assumed behavior accurately describes their end-project solution path. 


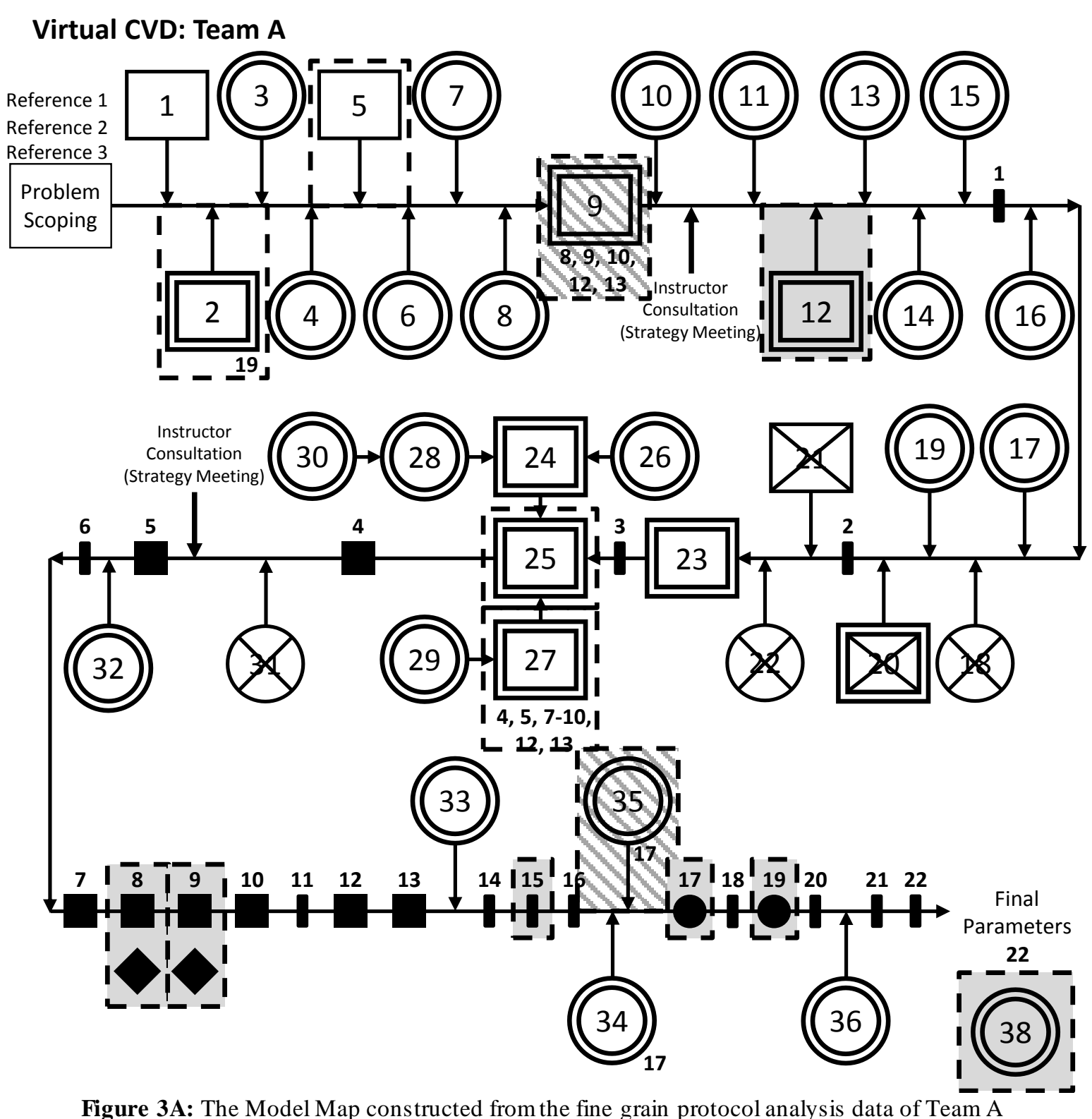

\section{General Observations}

The general interpretation of solution strategy utilized by Team A did not significantly change between the two data sources. Initial research quickly gave way to an integrated group of utilized model components. The primary group of model components was improved upon and the result leads to a short period of optimization via fine tuning.

This team's fine grain Model Map shows no model components which were 'not engaged', as opposed to the course grain Model Map showing one 'not engaged' model component. This could indicate the tendency for students to deliberate the usefulness of a model component verbally before making any indication in their notebook. Only one 'abandoned' model component is present in the course grain Model Map, while seven are present in the fine grain Model Map. 


\section{Virtual CVD: Team A}

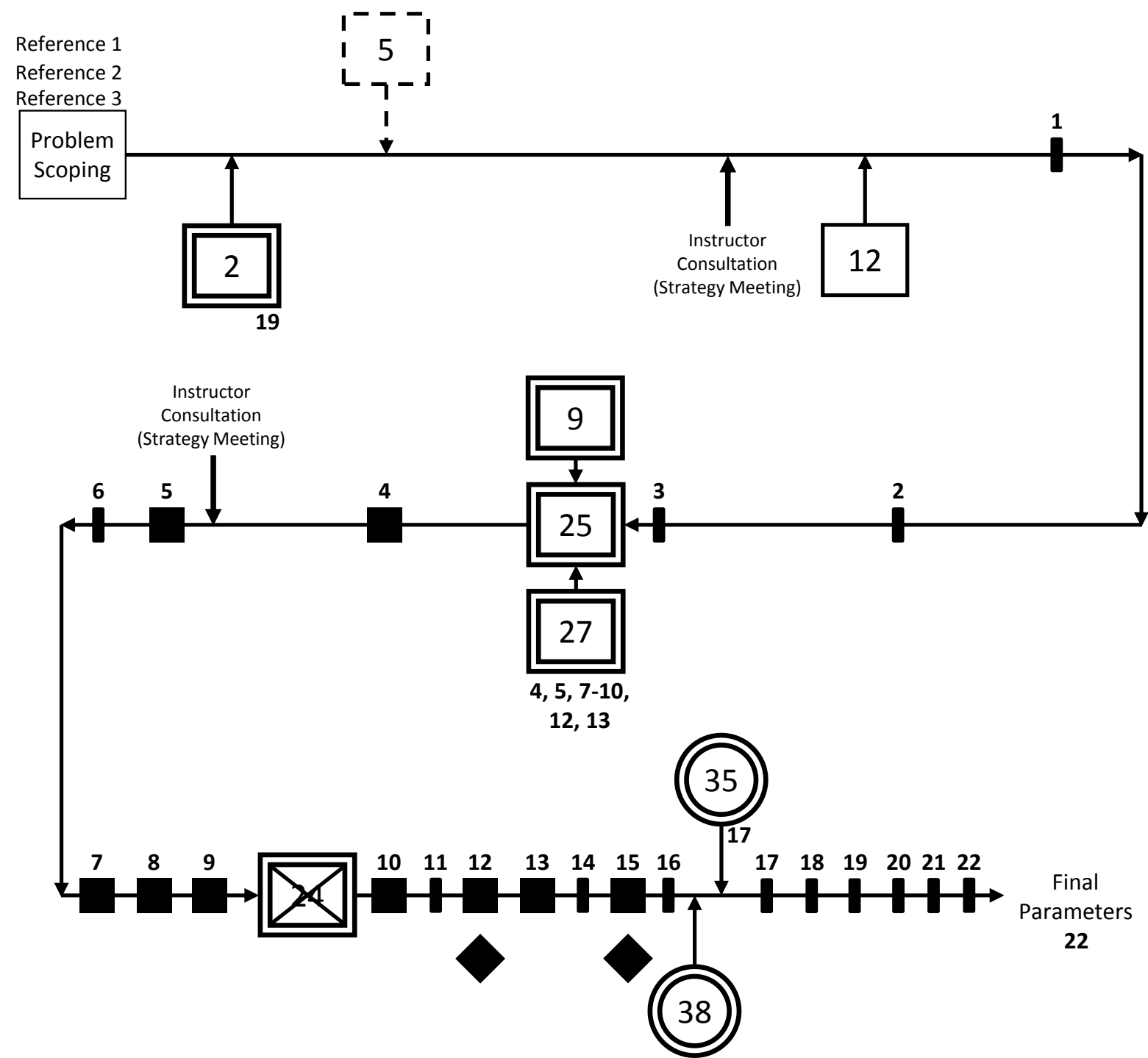

Figure 3B: The Model Map for Team A which depicts the course grain model components (from Figure 1A) with respect to the adjusted timeline created by the fine grain Model Map. The model components have also been renumbered to be consistent with the other Model Map.

\section{Protocol Analysis: Team B}

The Model Map from the fine grained data source for Team B is shown in Figure 4B. The components from the course grained data source (originally shown in Figure 1B) are redrawn in Figure $4 \mathrm{~B}$ with the length of the time line and the numbering changed to match Figure $4 \mathrm{~A}$. The level of detail also increase with the fine grain analysis of Team B. However, the interpretation of the problem formulation stage remains essentially the same. Team B shows a tendency to leave many ideas unconsidered. 


\section{Virtual CVD: Team B}
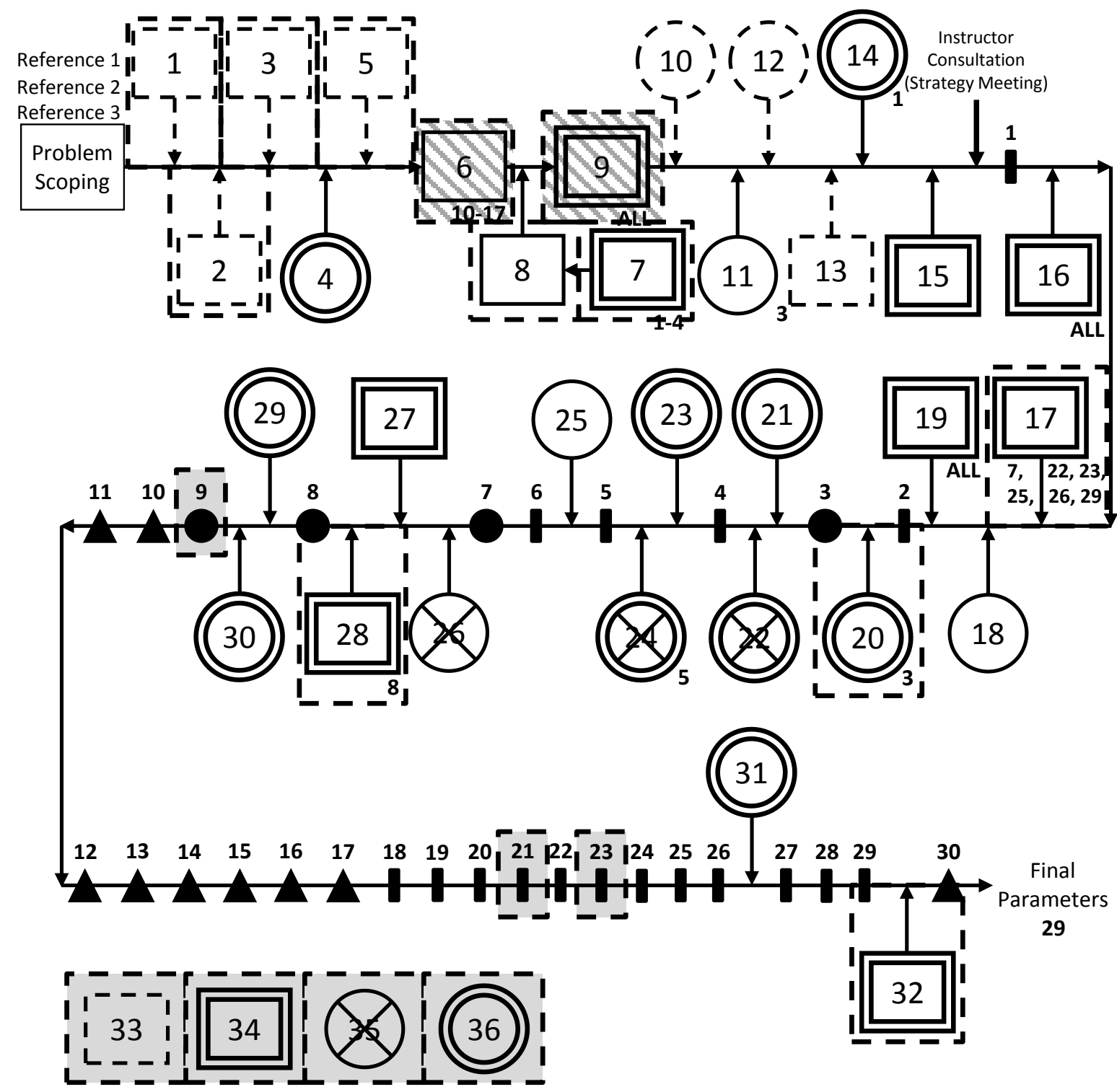

Figure 4A: The Model Map constructed from the fine grain protocol analysis data of Team B.

Statistical Methods and Qualitative Modeling

The first reference to an essential model component for the statistical approach appears much earlier in the fine grain Model Map (component 6) than the course grain Model Map; however, it is utilized in the same range of runs as it was in the notebook-based Model Map, except for run 21. In addition to the movement of this component, the qualitative modeling behavior, which was observed twice in the course grain Model Map, is shown in much greater depth via the 10 qualitative model components present between run 1 and run 9 . 
Virtual CVD: Team B

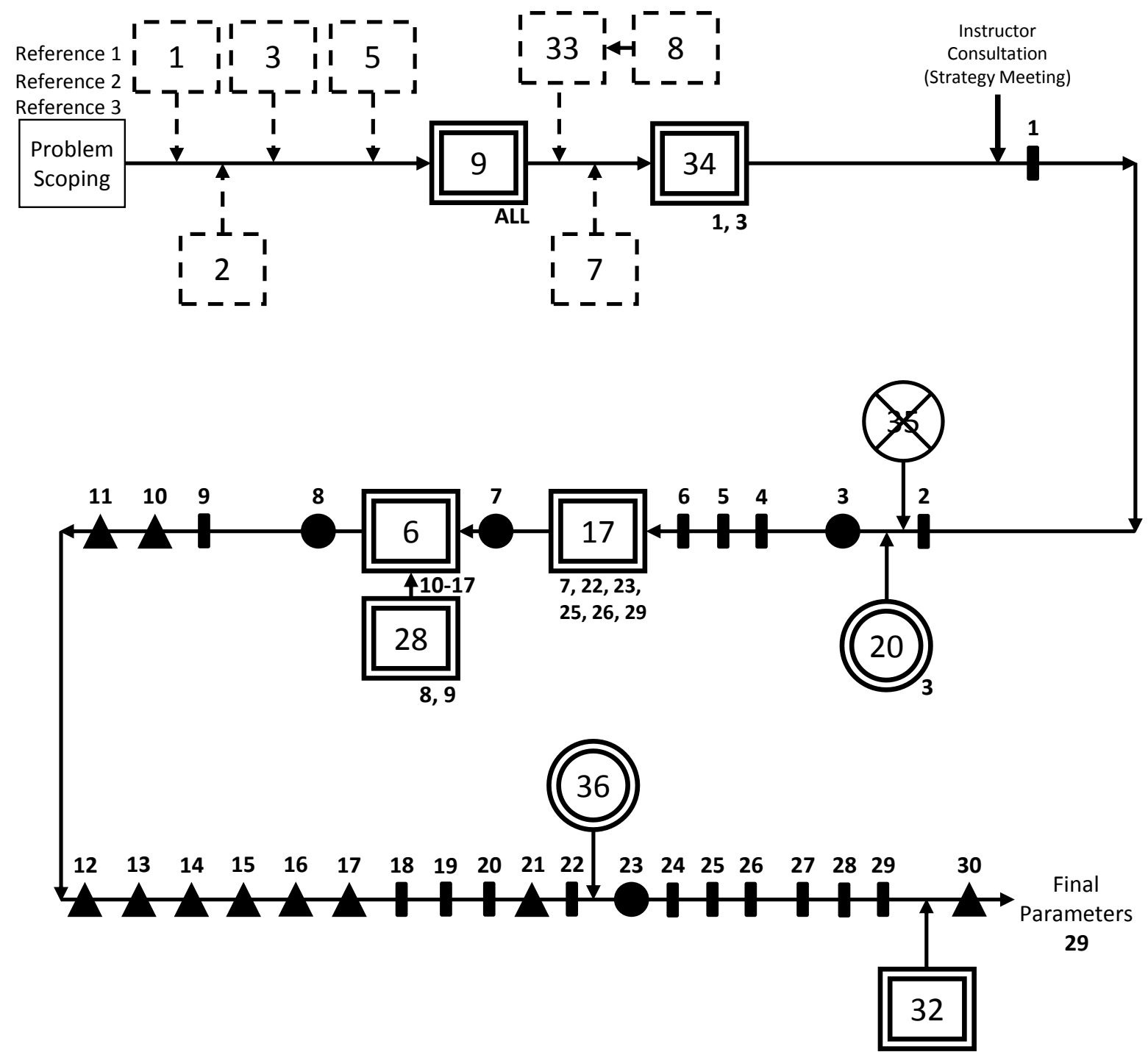

Figure 4B: The Model Map for Team B which depicts the course grain model components (from Figure 1B) with respect to the adjusted timeline created by the fine grain Model Map. The model components have also been renumbered to be consistent with the other Model Map.

Fine Tuning

Though not as extensive as for Team A, Team B's Model Map shows additional model components are present after the team moves on to their fine tuning phase (after run 17).

\section{General Observations}

Again the general interpretation of solution strategy does not significantly change between the two data sources. In both Model Maps, Team B starts the project with a scattered problem formulation effort which was followed by a string of qualitative model components. Their solution pathway focused when the statistical model component was implemented and their project was completed soon after their fine tuning runs were complete. 


\section{Consistency between Data Sources}

As was illustrated by Figure 3, a model component can change identity, undergo a movement, or remain unchanged. Similarly a changed run indicates that the interpretation of modeling activity associated with that run has changed. Based on this type of characterization, we have compared the modeling activity associated with runs and the type of model components between the course and fine grain data sources. Table 3 summarizes the results. Based on the fine grain analysis, approximately $5 \%$ of the model components moved and $18 \%$ of the runs or model components changed. The remaining $77 \%$ are consistent between the two data sources.

Table 3: Summary of Differences between Notebook and Protocol Analysis Model Maps

\begin{tabular}{|l|c|c|}
\hline & Team A & Team B \\
\hline Runs & 24 & 30 \\
\hline Model Components & 9 & 15 \\
\hline Total & 33 & 45 \\
\hline Runs Changed & 5 & 3 \\
\hline Model Components Moved & 2 & 2 \\
\hline Model Components Changed & 2 & 4 \\
\hline Percent Moved & \multicolumn{3}{|c|}{$5 \%$} \\
\hline Percent Changed & \multicolumn{3}{|c|}{$18 \%$} \\
\hline
\end{tabular}

\section{Summary and Future Work}

Model Maps based on data sources at two different grain sizes are compared in this study. While the fine grain size represented by "think-aloud" protocol analysis shows more detail, the essential features of the those developed by the coarser grain data source (work products) remains intact. The story line between the two sources is consistent and around $77 \%$ of the model components and runs are identical. This analysis suggests that Model Maps developed from student work products reasonably characterize teams in the Virtual CVD Laboratory Project. Performing analysis at the coarser grain size is advantageous for allowing a greater scope of analysis.

Moreover, the teams analyzed in this study are those few that participated in protocol analysis. We believe these teams may represent worst case scenarios. As a member from Team A stated, "We don't write much in our lab notebook because we totally feel we are communicating to the recorder." This utterance implies that the think aloud process has a negative effect on the detail in the notebook. In turn, the notebooks for teams that did not participate in the think aloud process should be a stronger indication of their parent groups' solution process.

While the fine grain Model Maps used to validate the course grain Model Maps in the work presented here; we believe that with modification, Model Maps based on protocol analysis can also be useful to characterize a critical element of the teams' modeling behavior. As they were developed for this paper, the Model Maps displayed each model component only once, at 
inception. However, we noticed many of these model components actually repeated themselves over the course of the project. Hence, we may next ask research questions such as:

1. Does the finer grain size of protocol analysis afford opportunity to observe the iterative cycle of creation, use, evaluation, and revision of model components that cannot be resolved at the coarser grain size?

2. What is the nature of the iterative cycle of modeling that manifests in the project?

\section{Acknowledgements}

The authors are grateful for support provided by the Intel Faculty Fellowship Program and the National Science Foundation's Course, Curriculum and Laboratory Improvement Program, under Proof-of-Concept grant DUE-0442832 and Phase 2 grant DUE-0717905. Any opinions, findings, and conclusions or recommendations expressed in this material are those of the authors and do not necessarily reflect the views of the National Science Foundation.

\section{References}

1. Gainsburg, J. 2006. The Mathematical Modeling of Structural Engineers. Mathematical Thinking and Learning. 8:1, 3-36.

2. Cardella, M. E., 2007. Mathematical Modeling in Engineering Design Projects: Insights from an Undergraduate Capstone Design Project and a Year-Long Graduate Course. Proceedings of the Thirteenth International Conference on the Teaching of Mathematical Modeling and Applications. Bloomington, Indiana.

3. Buckley, B., J. Gobert, P. Horwitz, \& L. O’Dwyer. 2010. Looking Inside the Black Box: Assessing ModelBased Learning and Inquiry in BioLogica. International Journal ofLearning Technology. 5(2), 166-189.

4. Koretsky, M.D., C. Kelly, \& E. Gummer. 2011. Student Perceptions of Learning in the Laboratory: Comparison of Industrially Situated Virtual Laboratories to Capstone Physical Laboratories. Journal of Engineering Education. 100(3), 540-573.

5. Koretsky, M.D., D. Amatore, C. Barnes, \& S. Kimura. 2008. Enhancement of Student Learning in Experimental Design using a Virtual Laboratory. IEEE Transactions on Education. 51 (1), 76-85.

6. Nefcy, E., P. Harding \& M. Koretsky. 2011. Characterization of Student Model Development in Physical and Virtual Laboratories. Proceedings of the 2011 American Society for Engineering Education Annual Conference \& Exposition.

7. D'Angelo, C., D.W. Shaffer, \& N. Chesler. 2011. Undergraduate Engineers Engaging and Reflecting in a Professional Practice Simulation. Proceedings of the 2011 American Society for Engineering Education Annual Conference \& Exposition .

8. Honey M. A., \& Hilton M (ed). 2011. Learning science through computer games and simulations. Washington, D.C: The National Academies Press.

9. Wiesner, T., \& W. Lan. 2004. Comparis on of student learning in physical and simulated unit operations experiments. Journal of Engineering Education, 93 (3), 195-204.

10. Campbell, J.O., J.R. Bourne, P.J. Mosterman, \&A.J. Brodersen. 2002. The effectiveness of learning simulations for electronic laboratories. Journal of Engineering Education.91 (1), 81-87.

11. Finklestein, N.D., W.K. Adams, C.J. Keller, P.B. Kohl, K.K. Perkins, N.S. Podolefsky, S. Reid and R. LeMaster. 2005. When learning about the real world is better done virtually: A study of substituting computer simulations for laboratory equipment. Physical Review Special Topics - Physics Education Research (1), 1-7.

12. Lindsay, E., \& M. Good. 2005. Effects of laboratory access nodes upon learning outcomes. IEEE Transactions on Education. 48 (4), 619-631. 
13. Zacharia, Z. 2007. Comparing and combining real and virtual experimentation: An effort to enhance students' conceptual understanding of electrical circuits. Journal of Computer Assisted Learning, 23 (2), 120-132.

14. Day, R.S. 2006. Challenges of biological realism and validation in simulation-based medical education. Artificial Intelligence in Medicine. 38(1): 47-66.

15. Hmelo C.E., S. Ramakrishnan, R.S. Day, W.E. Shirey, Q. Huang, and J. Baar. 1998. Developing inquiry skills through scaffolded use of a simulation. In International Conference of the Learning Sciences: Proceedings of the 1998 Conference. Charlottes ville, VA: Association for the Advancement of Computing in Education.

16. Atman, C. J., R.S. Adams, M.E. Cardella, J. Turns, S. Mosborg, \& J. Saleem. 2007. Engineering design processes: A comparison of students and expert practitioners. Journal of Engineering Education.96(4), 359.

17. Strom, D., V. Kemeny, R. Lehrer, \& E. Forman. 2001. Visualizing the emergent structure of children's mathematical argument. Cognitive Science, 25, 733-774.

18. Hmelo-Silver, C.E. 2003. Analyzing collaborative knowledge construction: Multiple methods for integrated understanding. Computers \& Education. 41: 397-420.

19. Hmelo-Silver, C.E., E. Chernobilsky, \& R. Jordan. 2008. Understanding collaborative learning processes in new learning environments. Instructional Science. 36(5), 409-430.

20. Hmelo-Silver, C.E., L. Liu, \& R. Jordan. 2009. Visual representation of a multidimensional coding scheme for understanding technology-mediated learning about complex natural systems. Research and Practice in Technology Enhanced Learning . 4(3) 253-280.

21. Shaffer, D.W., D. Hatfield, G.N. Svarovsky, P. Nash, A. Nulty, E. Bagley, K. Franke, A.A. Rupp, \& R. Mislevy. 2009. Epistemic Network Analysis: A prototype for 21st Century assessment of learning. The International Journal of Learning and Media. 1(2), 33-53.

22. Schwarz, C.V., B.J. Reiser, E.A. Davis, L.O. Kenyon, A. Acher, D. Fortus, Y. Shwartz, B. Hug, \& J. Krajcik. 2009. Developing a Learning Progression for Scientific Modeling: Making Scientific Modeling Accessible and Meaningful for Learners. Journal of Research in Science Teaching . 46 (6), 632-654.

23. Harrison, A.G. \& D.F. Treagust. 2010. A Typology of School Science Models. International Journal of Science Education. 22(9), 1011-1026.

24. Greca, I.M. \& M. A. Moreira. 2001. Mental, Physical, and Mathematical Models in the Teaching and Learning of Physics. Science Education. 86:1.

25. Dixon, R.A. 2010. Experts and Novices: Differences in Their Use of Mental Representation and Metacognition in Engineering Design. Ph.D. Thesis. University of Illinois at Urbana-Champaign.

26. diSessa, A.. 1993. Toward and Epistemology of Physics. Cognition and Instruction. 10(2 \& 3), 105-225.

27. Seniow, K., E. Nefcy, C. Kelly, \& M. Koretsky. 2010. Representations of Student Model Development in Virtual Laboratories based on a Cognitive Apprenticeship Instructional Design. Proceedings of the 2010 American Society for Engineering Education Annual Conference \& Exposition. 